\title{
308.
}

\section{ON THE $\triangle$ FACED POLYACRONS, IN REFERENCE TO THE PROBLEM OF THE ENUMERATION OF POLYHEDRA.}

[From the Memoirs of the Literary and Philosophical Society of Manchester, vol. I. (1862), pp. 248-256.]

THE problem of the enumeration of polyhedra $\left(^{1}\right)$ is one of extreme difficulty, and I am not aware that it has been discussed elsewhere than in $\mathrm{Mr}$ Kirkman's valuable series of papers on this subject in the Memoirs of the Society and in the Philosophical Transactions. A case of the general problem is that of the enumeration of the polyhedra with trihedral summits; and $\mathrm{Mr}$ Kirkman in the earliest of his papers, viz, that "On the representation and enumeration of polyhedra" (Memoirs, vol. XII. pp. 47-70, 1854), has in fact, by an examination of the particular case, accomplished the enumeration of the octahedra with trihedral summits. A subsequent paper "On the enumeration of $x$-edra having trihedral summits and an $(x-1)$ gonal base," Phil. Trans. vol. XLVI. pp. 399-411, 1856), relates, as the title shows, only to a special case of the problem of the polyhedra with trihedral summits, and in this particular case the number of polyhedra is more completely determined; but the later memoirs relate to the problem in all its generality, and the above-mentioned particular problem of the enumeration of the polyhedra with trihedral summits is not, I think, any where resumed. Instead of the polyhedra with trihedral summits, it is really the same thing, but it is rather more convenient to consider the polyacrons with triangular faces, or as these may for shortness be called, the $\Delta$ faced polyacrons; and it is intended in the present paper to give a method for the derivation of the $\Delta$ faced polyacrons of a given number of summits from those of the next inferior number of summits, and to exemplify it by finding, in an orderly manner, the $\Delta$ faced polyacrons

1 I use with Mr Kirkman the expression "enumeration of polyhedra" to designate the general problem, but I consider that the problem is to find the different polyhedra rather than to count them, and I consequently take the word enumeration in the popular rather than the mathematical sense. 
up to the octacrons: thus, as regards the examples, stopping at the same point as Mr Kirkman, for although perfectly practicable it would be very tedious to carry them further, and there would be no commensurate advantage in doing so. The epithet $\triangle$ faced will be omitted in the sequel, but it is to be understood throughout that I am speaking of such polyacrons only; and I shall for convenience use the epithets tripleural, tetrapleural, \&c. to denote summits with three, four, \&c. edges through them. The number of edges at a summit is of course equal to the number of faces, but it is the edges rather than the faces which have to be considered.

An $n$-acron has

$$
n \text { summits, } 3 n-6 \text { edges, } 2 n-4 \text { faces, }
$$

and it is easy to see that there are the following three cases only, viz. :

1. The polyacron has at least one tripleural summit.

2. The polyacron, having no tripleural summit, has at least one tetrapleural summit.

3. The polyacron, having no tripleural or tetrapleural summit, has at least twelve pentipleural summits.

In fact, if the polyacron has $c$ tripleural summits, $d$ tetrapleural summits, $e$ pentipleural summits, and so on, then we have

and therefore

$$
\begin{aligned}
n & =c+d+e+f+g+h+\& c \\
6 n-12 & =3 c+4 d+5 e+6 f+7 g+8 h+\& c
\end{aligned}
$$

or

$$
12=3 c+2 d+e+0 f-g-2 h-\& c .,
$$

$$
3 c+2 d+e=12+g+2 h+\& c \text {; }
$$

whence if $c=0$ and $d=0$, then $e=12$ at least. It appears, moreover (since $n$ cannot be less than $e$ ), that any polyacron with less than 12 summits cannot belong to the third class, and must therefore belong to the first or the second class.

An $(n+1)$-acron, by a process which I call the subtraction of a summit, may be reduced to an $n$-acron; viz., the faces about any summit of the $(n+1)$-acron stand upon a polygon (not in general a plane figure) which may be called the basic polygon, and when the summit with the faces and edges belonging to it is removed, the basic polygon, if a triangle, will be a face of the $n$-acron; if not a triangle, it can be partitioned into triangles which will be faces of the $n$-acron. The annexed figures exhibit the process for the cases of a tripleural, tetrapleural and pentipleural summit respectively, which are the only cases which need be considered; these may be called the first, second and third process respectively. It is proper to remark that for the same removed summit the first process can be performed in one way only, the second process in two ways, the third in five ways; these being in fact the numbers of ways of partitioning the basic polygon.

We may in like manner, by the converse process of the addition of a summit, convert an $n$-acron into an $(n+1)$-acron; viz., it is only necessary to take on the 
$n$-acron a polygon of any number of sides, and make this the basic polygon of the new summit of the $(n+1)$-acron, and for this purpose to remove the faces within the polygon and substitute for them a set of triangular faces standing on the sides of the polygon and meeting in the new summit: the same figures exhibit the process for the cases of a tripleural, tetrapleural and pentipleural summit respectively, which
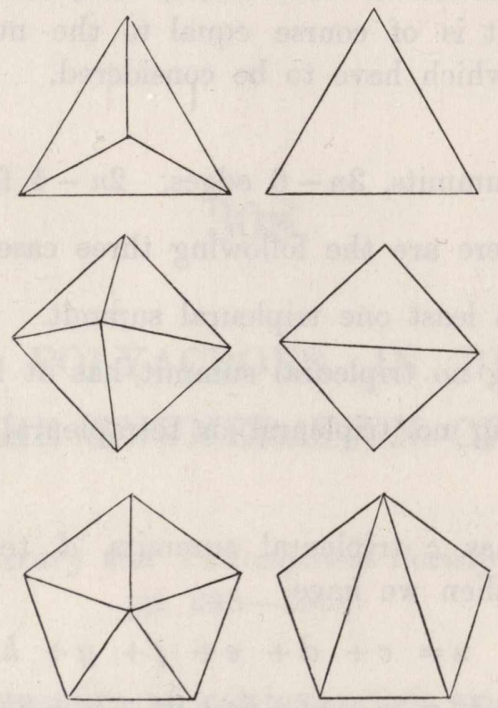

(as for the subtractions) are the only cases which need be considered. It may be noticed that for the same basic polygon the process is in each case a unique one; the process is said to be the first, second, or third process, according as the new summit is tripleural, tetrapleural, or pentipleural.

Now, reverting to the before-mentioned division of the polyacrons into three classes, an $(n+1)$-acron of the first class may by the first process of subtraction be reduced to an $n$-acron, and conversely it can be by the first process of addition derived from an $n$-acron. An $(n+1)$-acron of the second class, as having a tetrapleural summit, may by the second process of subtraction be reduced to an $n$-acron, and conversely it can be by the second process of addition derived from an $n$-acron. And in like manner, an $(n+1)$-acron of the third class, as having a pentipleural summit, may be by the third process of subtraction reduced to an $n$-acron, and conversely it may be by the third process of addition derived from an $n$-acron.

Hence all the $(n+1)$-acrons can be by the first, second and third processes of addition respectively derived from the $n$-acrons. It is to be observed that all the $(n+1)$-acrons of the first class are obtained by the first process; the second process is only required for finding the $(n+1)$-acrons of the second class; and these being all obtained by means of it, the third process is only required for finding the $(n+1)$-acrons of the third class. Hence the second process need only be made use of when the $n$-acron has no tripleural summit, or when it has only one tripleural summit, or when, having two tripleural summits, they are the opposite summits of two 
adjacent faces. In the last-mentioned two cases respectively it is only necessary to consider the basic quadrangles which pass through the single tripleural summit and the basic quadrangle which passes through the two tripleural summits; for with any other basic quadrangle the derived $(n+1)$-acron would retain a tripleural summit, and would consequently be of the first class. The condition is more simply expressed as follows, viz.: The second process need only be employed when there is on the $n$-acron a basic quadrangle the summits of which are at least of the number of edges shown in the

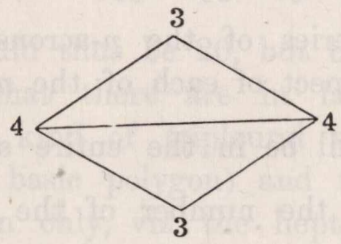

annexed nigure, and all the other summits are at least 4-pleural. Again, by the third process (as already mentioned) we seek only to obtain the $(n+1)$-acrons of the third class; the process need only be applied to the $n$-acrons for which there exists a basic pentagon the summits of which are at least of the number of edges shown in the

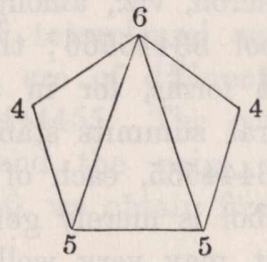

annexed figure, all the other summits being at least 5-pleural; for it is only in this case that the derived $(n+1)$-acron will be of the third class. The condition just referred to obviously implies that the $n$-acron is of the second or third class. It is to be noticed that in applying the foregoing principles to the formation of the polyacrons as far as the 11-acrons we are only concerned with the first and second processes.

Consider the entire series of $n$-acrons, say $A, B, C$, \&c., and suppose that the $n$-acron $A$ gives rise to a certain number, say $P, Q, R, S$ of $(n+1)$-acrons, the $(n+1)$ acron $P$ is of course derivable from the $n$-acron $A$, but it may be derivable from other $n$-acrons, suppose from the $n$-acrons $B$ and $C$. Then in considering the $(n+1)$ acrons derived from $B$, one of these will of course be found to be the $(n+1)$-acron $P$, and it is only the remaining $(n+1)$-acrons derived from $B$ which are or may be $(n+1)$-acrons not already previously obtained as $(n+1)$-acrons derived from $A$. And if in this manner, as soon as each $(n+1)$-acron is obtained, we apply to it the process of subtraction so as to ascertain the entire series of $n$-acrons from which it is derivable, and, in forming the $(n+1)$-acrons derived from these, take account of the $(n+1)$-acrons already previously obtained and found to be derivable from these, we should obtain without any repetitions the entire series of the $(n+1)$-acrons.

c. $v$. 
For merely finding the number of the $(n+1)$-acrons, a more simple process might be adopted: say that an $n$-acron is $p$-wise generating when it gives rise to a number $p$ of $(n+1)$-acrons, and that it is $q$-wise generable when it can be derived from a number $q$ of $(n+1)$-acrons; and assume that a given $n$-acron is $\left(y_{1}+y_{2}+y_{3}+\&\right.$ c.)wise generating, viz. that it gives rise to a number $y_{1}$ of $(n+1)$-acrons which are 1-wise generable, a number $y_{2}$ of $(n+1)$-acrons which are 2-wise generable, and so on; these forming the sum

$$
\Sigma\left(y_{1}+\frac{1}{2} y_{2}+\frac{1}{3} y_{3}+\ldots\right)
$$

where $\Sigma$ refers to the entire series of the $n$-acrons, it is clear that every $m$-wise generable $(n+1)$-acron will in respect of each of the $n$-acrons from which it is derivable be reckoned as $\frac{1}{m}$, that is, it will be in the entire sum reckoned as 1 , and the sum in question will consequently be the number of the $(n+1)$-acrons.

The figures of the polyacrons comprised in the annexed Tables show the application of the method to the genesis of the polyacrons as far as the octacrons, in which the numbers indicate the nature of the different summits, according to the number of edges through each summit, viz., 3 a tripleural summit, 4 a tetrapleural summit, and so on. It will be noticed that there is only a single case in which this notation is insufficient to distinguish the polyacron, viz., among the octacrons there are two forms each of them with the same symbol 33445566; the inspection of the figures shows at once that these are wholly distinct forms, for in the first of them, viz. that derived from 3344555 , each of the tripleural summits stands upon a basic triangle 456 , while in the other of them, that from 3444555, each of the tripleural summits stands upon a basic triangle 566. But the symbol is merely generic, and of course in the polyacrons of a greater number of summits it may very well happen that a considerable number of polyacrons are comprised in the same genus.

The following remarks on the derivation of the octacrons from the heptacrons will further illustrate the method:

1. The heptacron 3335556 has three kinds of faces, viz. $\left.355{ }^{1}\right), 356,555$, the first process consequently gives rise to 3 octacrons. As the heptacron has more than two tripleural summits the second process is not applicable.

2. The heptacron 3344466 has three kinds of faces, viz.: 366,346 and 446 , and the first process gives therefore 3 octacrons. The heptacron has only two tripleural summits, and they are disposed in the proper manner; the second process gives therefore 1 octacron.

3. The heptacron 3344556 has five kinds of faces, viz. $345,346,356,456$ and 4.55 , and the first process consequently gives 5 octacrons. The heptacron has two tripleural summits, but they are not disposed in such manner as to render the second process applicable.

${ }^{1}$ It is hardly necessary to remark that it must not be imagined that in general all the faces denoted by a symbol such as 355 (which determines only the nature of the summits on the face) are faces of the same kind, but this is so in the cases referred to in the text. 
4. The heptacron 3444555 has four kinds of faces, viz. $355,455,445$ and 444 , and the first process gives therefore 4 octacrons. The heptacron has one tripleural summit, and the basic quadrangles 3545 which belong to it are of the same kind; the second process gives therefore 1 octacron.

5. The heptacron 4444455 has only one kind of face, viz. 445 , and the first process gives therefore 1 octacron. There are two kinds of basic quadrangles, viz. 4545 and 4445 , and the second process gives therefore 2 octacrons.

The number of octacrons would thus be 20 , but by passing back from the octacrons to the heptacrons, it is found that there are in fact only 14 octacrons. Thus the octacron 33336666 has only one kind of tripleural summit 666 (the summit is here indicated by the symbol of the basic polygon) and the octacron is thus seen to be derivable from a single heptacron only, viz. the heptacron 3335556 from which it was in fact derived. But the octacron 33345567 has three kinds of tripleural summits, viz. 567,557 and 467, and it is consequently derivable from three heptacrons, viz. the heptacrons 3335556, 3344466 and 3344555, and so on. The passage to the heptacrons from an octacron with one or more tripleural summits is of course always by the first process, but for the last two octacrons, which have no tripleural summits, the passage back to the heptacrons is by the second process: thus for the octacron 44445555 we have but one kind of tetrapleural summit 4555 ; but as opposite pairs of summits of the basic quadrangle are of different kinds, viz. 45 and 55 , we obtain two heptacrons, viz. 3444555 and 4444455. The octacron 44444466 has but one kind of tetrapleural summit, viz. 4646, and the pairs of opposite summits of the basic quadrangle being of the same kind 46 , we obtain from it only the heptacron 4444455 .

It may be remarked that for the five heptacrons respectively the values of the sum $y_{1}+\frac{1}{2} y_{2}+\frac{1}{3} y_{3}+\ldots$ are

$$
1+\frac{1}{3}+\frac{1}{2}, \quad \frac{1}{3}+1+\frac{1}{2}+\frac{1}{2}, \quad \frac{1}{3}+\frac{1}{2}+\frac{1}{2}+1+1, \quad 1+1+1+1+\frac{1}{2}, \quad 1+\frac{1}{2}+\frac{1}{2},
$$

giving for $\Sigma\left(y_{1}+\frac{1}{2} y_{2}+\frac{1}{3} y_{3}+\ldots\right)$ the value 14 , as it should do. 